\title{
Two-pole Caustic Model for High-energy Radiation from Pulsars - Polarization
}

\author{
Jarosław Dyks, Alice K. Harding \\ Laboratory for High Energy Astrophysics, NASA/GSFC, Greenbelt, MD \\ 20771, USA \\ Bronisław Rudak \\ Nicolaus Copernicus Astronomical Center, 87-100 Toruń, Poland
}

\begin{abstract}
We present high-energy light curves and polarization properties of pulsars calculated within the recently proposed two-pole caustic model. The results are compared with the optical data for the Crab pulsar and with predictions of other high-energy pulsar models: the polar cap model, and the outer gap model.
\end{abstract}

\section{The Model}

The two-pole caustic (TPC) model (Dyks \& Rudak 2003) assumes uniform photon emission from a thin region confined to the surface of the last open magneticfield lines, and extending over their entire length between the polar cap and the light cylinder. The dipolar magnetosphere and photon emission tangent to magnetic field lines are assumed.

\section{Polarization}

In the high-energy domain, pulsar polarization data are available exclusively for optical radiation of the Crab pulsar (Smith et al. 1988; Kanbach et al. 2003). The left column of Figure 1 presents the light curve, the position angle (PA) curve, and the degree of polarization for the Crab pulsar obtained with the OPTIMA instrument (Kanbach et al. 2003). Two fast swings of PA and very low polarization degree at both peaks are noticeable.

The light curves and polarization characteristics calculated within the TPC, the polar cap, and the outer gap models are shown in the other columns of Figure 1. The PA and the degree of polarization were calculated assuming that the electric vector is parallel to the acceleration of the electron at the emission point. The TPC model predicts fast swings of the PA and minima in the polarization degree at both peaks which resemble those observed in the optical band for the Crab pulsar. Although exact agreement cannot be achieved under present assumptions, the approximate anticorrelation between the received flux and the polarization degree is noticeable, and in line with the Crab data. Contrary to TPC, neither the outer gap model nor the polar cap model are able to reproduce 

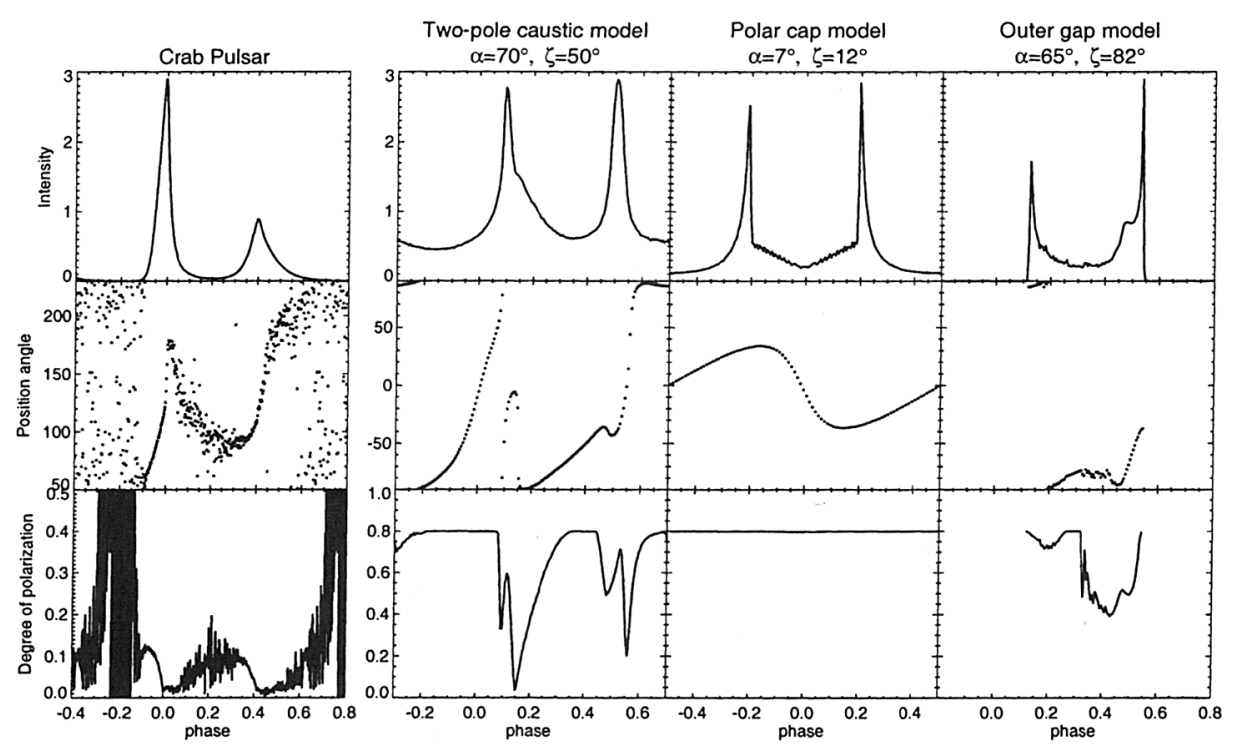

Figure 1. Left column: The optical light curve (top panel), the position angle curve (middle panel) and the degree of polarization (bottom panel) observed for the Crab pulsar by OPTIMA (Kanbach et al. 2003; Kellner 2002). Three right-hand side columns: the same radiation characteristics for the Crab pulsar as in the left column, but calculated with the three models of high energy radiation from pulsars: the two-pole caustic model, the polar cap model, and the outer gap model.

the optical polarization data on the Crab (we do not confirm the previous outer gap polarization result presented in Fig. 5 of Romani \& Yadigaroglu 1995). Detailed discussion of polarization results will be presented in Dyks, Harding \& Rudak (in preparation).

Acknowledgments. This work was performed while JD held a National Research Council Research Associateship Award at NASA/GSFC. This work was also supported by the grant 2P03D.004.24 (JD and BR) and by the NASA Astrophysics Theory Program (AH).

\section{References}

Dyks, J., \& Rudak, B. 2003, ApJ, 598, 1201

Kanbach, G., Kellner, S., Schrey, F. Z., Steinle, H., Straubmeier, C., \& Spruit, H. C. 2003, in Proc. of the SPIE, eds. M. Iye, \& A. F. M. Moorwood, 4841, p. 82

Kellner, S. 2002, PhD thesis, Technische Universität München

Romani, R. W., \& Yadigaroglu, I.-A. 1995, ApJ, 438, 314

Smith, F. G., Jones, D. H. P., Dick, J. S. B., \& Pike, C. D. 1988, MNRAS, 233, 305 